\title{
Weak and Strong MHD Turbulence
}

\author{
Grigol Gogoberidze ${ }^{* \dagger}$, Swadesh Mahajan**, Stefaan Poedts* and Mariam \\ Akhalkatsi $^{\dagger}$ \\ ${ }^{*}$ Centre for Plasma Astrophysics, K.U.Leuven, Celestijnenlaan 200B, 3001 Leuven, Belgium \\ ${ }^{\dagger}$ Institute of Theoretical Physics, Ilia State University, 3/5 Cholokashvili str., 0162 Tbilisi, Georgia \\ ** Institute for Fusion Studies, University of Texas at Austin, Austin, TX 78712
}

\begin{abstract}
The general conditions for the weak and strong regimes of incompressible magnetohydrodynamic turbulence are derived and studied in the framework of the direct interaction approximation. It is shown that in the framework of the weak turbulence theory, the autocorrelation and cascade timescales are always of the same order of magnitude. This means that, contrary to the general belief, any model of turbulence which implies a large number of collisions among wave packets for an efficient energy cascade (such as the Iroshnikov-Kraichnan model), does not represent a model of weak turbulence.
\end{abstract}

Keywords: Magnetohydrodynamic turbulence

PACS: 52.35.Ra, 47.27.Gs, 47.27.Eq

\section{INTRODUCTION}

Magnetohydrodynamic (MHD) turbulence is present in a wide variety of astrophysical systems such as the solar wind, the interstellar medium, accretion discs, and so on. Incompressible MHD is the standard model for the study of astrophysical MHD turbulence. Although incompressible MHD turbulence has been intensively studied for the last several decades $[1,2,3,4,5,6,7,8,9,10,11,12,13,14,15,16,17,18]$ many physical aspects of the problem still remain unclear. The first model of incompressible MHD turbulence was proposed by Iroshnikov [1] and Kraichnan [2]. The Iroshnikov-Kraichnan (IK) model of MHD turbulence is based on the fact that nonlinear interaction is possible only among Alfvén waves propagating in opposite directions along the mean magnetic field. Therefore, an energy cascade occurs as a result of collisions between oppositely propagating Alfvén waves. Consider the isotropic excitation of Alfvén waves on some outer scale $l_{0}$ with a characteristic velocity $v_{0} \ll V_{A}$, where $V_{A}$ denotes the Alfvén velocity. The IK model assumes that the energy transfer is local and isotropic in the wave number space. The characteristic time scale of the Alfvén wave collision is $\tau_{a c}^{I K} \sim\left(k V_{A}\right)^{-1}$, where $k$ is the wave number. Using the governing equations of incompressible MHD, it can be shown that during one collision the distortion of each wave packet $\delta v_{l}$ is of the order $\delta v_{l} / v_{l} \sim v_{l} / V_{A} \ll 1$. Because these perturbations are summed with random phases $N \sim\left(v_{l} / \delta v_{l}\right)^{2} \sim\left(V_{A} / v_{l}\right)^{2}$, collisions are necessary to achieve the distortion of order unity. Therefore, for the energy cascade time $\tau_{\text {cas }}^{l K}$ we have

$$
\tau_{\text {cas }}^{l K} \sim \frac{1}{k v_{l}} \frac{V_{A}}{v_{l}} .
$$

Taking into account the relations $\varepsilon \sim v_{l}^{2} / \tau_{c a s}$ and $v_{l}^{2} \sim k \mathscr{E}_{k}$, where $\varepsilon$ is the energy cascade rate and $E_{k}$ is the one dimensional energy spectrum, we obtain

$$
\mathscr{E}_{k} I K \sim\left(\varepsilon V_{A}\right)^{1 / 2} k^{-3 / 2},
$$

which represents the IK spectrum of incompressible MHD turbulence. Due to the condition $v_{0} \ll V_{A}$ the IK model is usually deemed as the model of weak MHD turbulence (i.e., the model for which perturbation theory called weak turbulence theory (WTT) $[19,20]$ is applicable). In general, it is commonly believed that any heuristic model of MHD turbulence which implies $N \gg 1$ represents the model of weak turbulence, whereas the models with $N \sim 1$ are models of strong turbulence.

The IK model for MHD turbulence is isotropic. However, the presence of a mean magnetic field has a strong effect on the turbulence properties, in contrast to a mean flow in hydrodynamic turbulence, which can be eliminated by the corresponding Galilean transformation. The anisotropy of MHD turbulence had been seen in various numerical simulations $[3,8,10,12]$. Different theoretical models of weak $[5,7,9]$ as well as strong $[4,6,17,18]$ anisotropic MHD turbulence have been developed to the date. In Ref. [3] it was shown that the lowest order three wave interactions 
among Alfvén waves are possible only if the parallel (with respect to the constant magnetic field) wave number of one mode is zero. This implies that in the framework of WTT there is no parallel cascade of energy. Consequently, the turbulence is anisotropic and the energy is cascaded to larger values of the perpendicular wave number.

In the case of zero cross and magnetic helicities, the stationary spectrum of the weak MHD turbulence is usually derived based on arguments similar to those of the IK model: consider the isotropic excitation of Alfvén waves on some outer scale $l_{0} \sim 1 / k_{0}$ with a characteristic velocity $v_{0} \ll V_{A}$ at $l_{0}$. For counter propagating wave packets with typical perpendicular size $l_{\perp} \sim 1 / k_{\perp}$, the characteristic collision time is $\tau_{a c} \sim\left(1 / k_{0} V_{A}\right)$ because the parallel size of the wave packets remains constant. According to the Euler equation, the partial distortion of each wave packet is $\delta v_{l} / v_{l} \sim\left(k_{\perp} v_{l}\right) /\left(k_{0} V_{A}\right) \ll 1$. Introducing a three dimensional energy spectrum $E\left(k_{\|}, k_{\perp}\right)$ such that $\int E\left(k_{\|}, k_{\perp}\right) \mathrm{d}^{2} \mathbf{k}_{\perp} \mathrm{d} k_{\|}$ is the total energy of the perturbations and using the Kolmogorov hypothesis, similar to the IK model one obtains $E\left(k_{\|}, k_{\perp}\right) \sim \varepsilon^{1 / 2} k_{\perp}^{-3}$. As the cascade proceeds to larger $k_{\perp}$, the ratio $\left(k_{\perp} v_{l}\right) /\left(k_{0} V_{A}\right)$ increases and when it reaches the value of order unity the turbulence becomes strong. Hence, the validity criterium of the WTT for anisotropic MHD turbulence is [6]

$$
\frac{k_{\perp} v_{l}}{k_{\|} V_{A}} \ll 1
$$

The usual interpretation of this condition implies that the nonlinear strain time should be less then the wave period.

In the present paper we study incompressible MHD turbulence in the framework of the weak coupling approximation (WCA) [19], which represents one of the equivalent formulations of the direct interaction approximation (DIA) [22]. The WCA allows us to study both limits (weak and strong) of incompressible MHD turbulence in the framework of a unified formalism. We rederive the WTT equations from the WCA equations of incompressible MHD turbulence derived earlier in Ref. [9]. We then derive the general validity condition of the WTT, which reads $\tau_{a c} k_{\|} V_{A} \ll 1$, where $\tau_{a c}$ is the Lagrangian autocorrelation timescale. In the simplest case, this condition reduces to Eq. (3) but in more complicated cases there exist non trivial differences. We show that in the framework of the WTT the autocorrelation and cascade timescales are always of the same order of magnitude. This means that, contrary to the general belief, in the WTT $N \sim 1$ and, consequently, any model for MHD turbulence that implies $N \gg 1$ (such as the IK model or the heuristic model of anisotropic turbulence described above) does not represent the model of weak turbulence. We also consider the strong turbulent limit and show that if isotropy is assumed then the WCA equations automatically lead to the IK spectrum.

The paper is organized as follows. The WCA formalism for incompressible MHD turbulence is described in Sec. 2. The WTT equations are derived and analyzed in Sec. 3 and 4, respectively. The strong turbulence limit is considered in Sec. 5. The conclusions are given in Sec. 6 .

\section{THE WCA EQUATIONS FOR INCOMPRESSIBLE MHD TURBULENCE}

Consider incompressible MHD turbulence in the presence of a constant magnetic field $\mathbf{B}_{0}$ directed along $z$ axis. The equations of ideal MHD, governing the evolution of fluctuations of the Elsasser variables, are

$$
\begin{gathered}
\partial_{t} \mathbf{U}_{1}-\mathbf{V}_{A} \partial_{z} \mathbf{U}_{1}=-\left(\mathbf{W}_{1} \cdot \nabla\right) \mathbf{U}_{1}-\nabla p \\
\partial_{t} \mathbf{W}_{1}+\mathbf{V}_{A} \partial_{z} \mathbf{W}_{1}=-\left(\mathbf{U}_{1} \cdot \nabla\right) \mathbf{W}_{1}-\nabla p
\end{gathered}
$$

where $\mathbf{V}_{A} \equiv \mathbf{B}_{\mathbf{0}} / \sqrt{4 \pi \rho}$ is the Alfvén velocity, $\mathbf{W}_{1}=\mathbf{v}_{1}+\mathbf{b}_{1}$ and $\mathbf{U}_{1}=\mathbf{v}_{1}-\mathbf{b}_{1}$ are fluctuations of the Elsasser variables, $\mathbf{v}_{1}$ is the turbulent velocity field, $\mathbf{b}_{1} \equiv \mathbf{B}_{1} / \sqrt{4 \pi \rho}$ denotes the magnetic field of the fluctuations in velocity units, $p$ is the pressure normalized by the density and $\partial_{t} \equiv \partial / \partial t$.

Performing a Fourier transform defined as

$$
\mathbf{u}_{\mathbf{k}, \omega}=\frac{1}{(2 \pi)^{4}} \int \exp (i \omega t-i \mathbf{k} \cdot \mathbf{x}) \mathbf{U}_{1}(\mathbf{x}, t) d^{3} \mathbf{x} d t
$$

neglecting pseudo Alfvén waves which are known to play only a passive role [6], defining the unit polarization vector of the shear Alfvén waves as $\hat{\mathbf{e}}_{\mathbf{k}}=\hat{\mathbf{k}} \times \mathbf{z}$, and introducing the amplitudes of the shear Alfvén waves as

$$
\mathbf{w}_{\overline{\mathbf{k}}}=i \phi_{\overline{\mathbf{k}}} \hat{\mathbf{e}}_{\mathbf{k}}, \quad \mathbf{u}_{\overline{\mathbf{k}}}=i \psi_{\overline{\mathbf{k}}} \hat{\mathbf{e}}_{\mathbf{k}}
$$

Eqs. (4)-(5) reduce to the following set of equations

$$
\left(\omega-\omega_{\mathbf{k}}\right) \phi_{\overline{\mathbf{k}}}=\int_{-\infty}^{\infty} T_{1,2} \phi_{1} \psi_{2} d \mathscr{F}_{1,2}^{k}
$$




$$
\left(\omega+\omega_{\mathbf{k}}\right) \psi_{\overline{\mathbf{k}}}=\int_{-\infty}^{\infty} T_{1,2} \psi_{1} \phi_{2} d \mathscr{F}_{1,2}^{k}
$$

where $T_{1,2} \equiv i\left(\hat{\mathbf{e}}_{\mathbf{k}} \cdot \hat{\mathbf{e}}_{\mathbf{k}_{\mathbf{1}}}\right)\left(\mathbf{k} \cdot \hat{\mathbf{e}}_{\mathbf{k}_{\mathbf{2}}}\right)$ is the matrix element of interaction.

Applying the standard technique of WCA, one can then obtain the following set of equations [17]

$$
\begin{gathered}
\zeta_{\overline{\mathbf{k}}}^{+}=\int_{-\infty}^{\infty}\left|T_{1,2}\right|^{2} \Gamma_{1}^{+} I_{2}^{-} d \mathscr{F}_{1,2}^{k}, \\
\zeta_{\overline{\mathbf{k}}}^{-}=\int_{-\infty}^{\infty}\left|T_{1,2}\right|^{2} \Gamma_{1}^{-} I_{2}^{+} d \mathscr{F}_{1,2}^{k}, \\
-i\left(\omega-\omega_{\mathbf{k}}\right) I_{\overline{\mathbf{k}}}^{+}=\Gamma_{\overline{\mathbf{k}}}^{+*} \int_{-\infty}^{\infty}\left|T_{1,2}\right|^{2} I_{1}^{+} I_{2}^{-} d \mathscr{F}_{1,2}^{k}-I_{\overline{\mathbf{k}}}^{+} \int_{-\infty}^{\infty}\left|T_{1,2}\right|^{2} \Gamma_{1}^{+} I_{2}^{-} d \mathscr{F}_{1,2}^{k}, \\
-i\left(\omega+\omega_{\mathbf{k}}\right) I_{\overline{\mathbf{k}}}^{-}=\Gamma_{\overline{\mathbf{k}}}^{-*} \int_{-\infty}^{\infty}\left|T_{1,2}\right|^{2} I_{1}^{-} I_{2}^{+} d \mathscr{F}_{1,2}^{k}-I_{\overline{\mathbf{k}}}^{-} \int_{-\infty}^{\infty}\left|T_{1,2}\right|^{2} \Gamma_{1}^{-} I_{2}^{+} d \mathscr{F}_{1,2}^{k},
\end{gathered}
$$

where $\overline{\mathbf{k}} \equiv(\mathbf{k}, \omega)$, the caret denotes the unit vector, $\mathbf{u}_{1}$ denotes $\mathbf{u}_{\overline{\mathbf{k}}_{1}}, \omega_{\mathbf{k}}=k_{z} V_{A}$ is the frequency of the Alfvén wave, $d \mathscr{F}_{1,2}^{k} \equiv d^{4} \overline{\mathbf{k}}_{1} d^{4} \overline{\mathbf{k}}_{2} \delta_{\overline{\mathbf{k}}-\overline{\mathbf{k}}_{1}-\overline{\mathbf{k}}_{2}}$, and $\delta_{\overline{\mathbf{k}}-\overline{\mathbf{k}}_{1}-\overline{\mathbf{k}}_{2}} \equiv \delta\left(\overline{\mathbf{k}}-\overline{\mathbf{k}}_{1}-\overline{\mathbf{k}}_{2}\right)$ is the Dirac delta function,

$$
\left\langle\phi_{\overline{\mathbf{k}}} \phi_{\overline{\mathbf{k}}^{\prime}}^{*}\right\rangle=I_{\overline{\mathbf{k}}}^{+} \delta_{\overline{\mathbf{k}}-\overline{\mathbf{k}}^{\prime}}, \quad\left\langle\psi_{\overline{\mathbf{k}}} \psi_{\overline{\mathbf{k}}^{\prime}}^{*}\right\rangle=I_{\overline{\mathbf{k}}}^{-} \delta_{\overline{\mathbf{k}}-\overline{\mathbf{k}}^{\prime}}
$$

and

$$
\Gamma_{\overline{\mathbf{k}}}^{ \pm}=\frac{i}{\omega \mp \omega_{\mathbf{k}}+i \zeta_{\overline{\mathbf{k}}}^{ \pm}} .
$$

Eqs. (10)-(13) are useless unless some assumptions are made about the frequency dependence of $\Gamma_{\overline{\mathbf{k}}}^{ \pm}$and $I_{\overline{\mathbf{k}}}^{ \pm}$. Equivalently, in the framework of the DIA one should make some assumptions about the time dependence of $G^{ \pm}(\mathbf{k}, \tau)$ and $Q^{ \pm}(\mathbf{k}, \tau)$ [21], which are the corresponding inverse Fourier transforms of $\Gamma_{\overline{\mathbf{k}}}^{ \pm} / 2 \pi$ and $I_{\overline{\mathbf{k}}}^{ \pm}$with respect to $\omega$ [i.e., $\left.\left\langle\phi_{\mathbf{k}}(t+\tau) \phi_{\mathbf{k}^{\prime}}^{*}(t)\right\rangle=Q^{+}(\mathbf{k}, \tau) \delta_{\mathbf{k}-\mathbf{k}^{\prime}}\right]$. One of the simplest and frequently used assumptions implies [22, 21]

$$
\begin{gathered}
G^{ \pm}(\mathbf{k}, \tau)=\exp \left(-\left|\eta_{\mathbf{k}}^{ \pm}\right| \tau \pm i \omega_{\mathbf{k}} \tau\right) H(\tau), \\
Q^{ \pm}(\mathbf{k}, \tau)=\exp \left(-\left|\eta_{\mathbf{k}}^{ \pm}\right| \tau \pm i \omega_{\mathbf{k}} \tau\right) E_{\mathbf{k}},
\end{gathered}
$$

where $H(t)$ is the Heaviside (step) function, and $E_{\mathbf{k}}$ is the energy spectrum.

Similar to Eqs. (16)-(17), in the case under consideration we assume

$$
\begin{gathered}
\zeta_{\overline{\mathbf{k}}}^{ \pm}=\eta_{\mathbf{k}}^{ \pm}, \\
I_{\overline{\mathbf{k}}}^{ \pm}=\frac{E_{\mathbf{k}}^{ \pm}}{\pi} \frac{\eta_{\mathbf{k}}^{ \pm}}{\left(\omega \mp \omega_{\mathbf{k}}\right)^{2}+\left(\eta_{\mathbf{k}}^{ \pm}\right)^{2}} .
\end{gathered}
$$

Here, for simplicity, we consider the symmetric case $\eta_{\mathbf{k}}^{ \pm}=\eta_{\mathbf{k}}$, and $E_{\mathbf{k}}^{ \pm}=E_{\mathbf{k}}$, which physically corresponds to a turbulence with zero cross helicity. Although we consider the symmetric case, in the further analysis we will keep the \pm signs for the energy spectra in order to underline the fact that nonlinear interactions are possible only between counter propagating modes.

$\tau_{a c} \equiv 1 / \eta_{\mathbf{k}}$ is the autocorrelation time. As it was mentioned in Refs. [19, 2] the random Galilean invariance requires that before applying the WCA (DIA) closure scheme to Eqs. (8)-(9) one should remove the influence of the velocity field of low frequency modes (for a more detailed analysis see Ref. [17]). If this is done, and the corresponding contributions are removed from Eqs. (10)-(11), then $\tau_{a c}$ represents the Lagrangian autocorrelation timescale, which is called "the duration of unit act of interaction" in heuristic models of the turbulence. Note that the fact that the correlation is lost at some specific point does not imply that correlated fluctuations ("wave packets") are decayed by nonlinear interactions. It just implies that the correlation is lost in some specific point, but they can still exist in an another point. The real "duration of life" of a specific wave packet characterizes the energy transfer between different scales. It is called the "cascade timescale", $\tau_{c a s}$, and can be determined by the energy balance equation (12) as the characteristic timescale of the process. 


\section{DERIVATION OF THE WTT EQUATIONS}

Substituting Eqs. (18) and (19) into Eq. (10) and performing an integration with respect to the frequencies, we get

$$
\begin{gathered}
-i\left(\omega-\omega_{\mathbf{k}}\right) I_{\overline{\mathbf{k}}}^{+}=\Gamma_{\overline{\mathbf{k}}}^{+*} \int_{-\infty}^{\infty}\left|T_{1,2}\right|^{2} R_{a} E_{1}^{+} E_{2}^{-} \mathrm{d} \mathscr{K}_{1,2}^{k}-I_{\overline{\mathbf{k}}}^{+} \int_{-\infty}^{\infty}\left|T_{1,2}\right|^{2} R_{b} E_{2}^{-} \mathrm{d} \mathscr{K}_{1,2}^{k}, \\
\eta_{\mathbf{k}}=\int_{-\infty}^{\infty}\left|T_{1,2}\right|^{2} R_{b} E_{2}^{-} \mathrm{d} \mathscr{K}_{1,2}^{k}
\end{gathered}
$$

where

$$
\begin{gathered}
R_{a}=\frac{1}{\pi} \frac{\eta_{1}+\eta_{2}}{\left(\eta_{1}+\eta_{2}\right)^{2}+\left(\omega-\omega_{k}+\Delta \omega_{k 12}\right)^{2}}, \\
R_{b}=\frac{1}{\left(\eta_{1}+\eta_{2}\right)-i\left(\omega-\omega_{k}+\Delta \omega_{k 12}\right)},
\end{gathered}
$$

$\mathrm{d} \mathscr{K}_{1,2}^{k} \equiv \mathrm{d}^{3} \mathbf{k}_{1} \mathrm{~d}^{3} \mathbf{k}_{2} \delta_{\mathbf{k}-\mathbf{k}_{1}-\mathbf{k}_{2}}$, and $\Delta \omega_{k 12}=\omega_{\mathbf{k}}-\omega_{\mathbf{k}_{1}}+\omega_{\mathbf{k}_{2}}$

According to Eq. (19), $I_{\overline{\mathbf{k}}}^{ \pm}$significantly differs from zero when $\left(\omega-\omega_{\mathbf{k}}\right)<\eta_{\mathbf{k}}$. First of all, consider the real part of the right hand side of Eq. (20) which describes the nonlinear decay of the fluctuations (whereas the real part describes the frequency shift caused by nonlinear interactions). Consider the limit $\eta_{\mathbf{k}} \rightarrow 0$. In this case, the width of the wave packets tends to zero and Eq. (19) yields $I_{\overline{\mathbf{k}}}^{ \pm}=E_{\mathbf{k}} \delta_{\omega \mp \omega_{\mathbf{k}}}$. In the considered limit the integrals on the right hand side of Eq. (20) are dominated by the contribution of the small vicinity of the so-called resonant curve (defined by the condition $\Delta \omega_{k 12}=0$ ) where the condition

$$
\Delta \omega_{k 12}<\eta_{\mathbf{k}}
$$

is fulfilled. The solution of the resonant condition $\Delta \omega_{k 12}=0$ is $k_{z 2}=0$. Consequently, three wave resonant interactions must include the zero frequency mode $[3,7,9]$. The volume of the wave number space occupied by the resonant area where the condition (24) is fulfilled is $\left[\eta_{\mathbf{k}} /\left(\partial \omega_{k} / \partial k_{z}\right)\right] k_{\perp}^{2}$. Taking also into account that a typical value of $R_{a}$ and $\Re\left(R_{b}\right) / \pi$ in the resonant area is $1 / \eta_{\mathbf{k}}$, noting that $T_{1,2} \sim k_{\perp}$ and assuming that the nonlinear energy transfer in the $\mathbf{k}_{\perp}$ plane is dominated by triad interactions with $k_{\perp} \sim k_{\perp 1,2}$ then the contribution of the resonant area in (say) the first integral of Eq. (20) can be estimated as $k_{\perp}^{4} E^{+}\left(k_{z}, k_{\perp}\right) E^{-}\left(0, k_{\perp}\right) /\left(\partial \omega_{k} / \partial k_{z}\right)$. Similarly, the contribution of the rest part of the $\mathbf{k}$ space is $\eta_{\mathbf{k}} k_{z} k_{\perp}^{4} E^{+}\left(k_{z}, k_{\perp}\right) E^{-}\left(k_{z}, k_{\perp}\right) / \omega_{k}^{2}$. Consequently, the domination of the resonant contribution implies

$$
\frac{\eta_{\mathbf{k}}}{\omega_{\mathbf{k}}} \frac{E^{-}\left(k_{z}, k_{\perp}\right)}{E^{-}\left(0, k_{\perp}\right)} \ll 1 .
$$

If this condition is fulfilled, one can replace $R_{a}$ and $\Re\left(R_{b}\right) / \pi$ by $\delta\left(\omega_{\mathbf{k}}-\omega_{\mathbf{k}_{1}}+\omega_{\mathbf{k}_{2}}\right) \equiv \delta_{\Delta \omega_{k 12}}$. Then, the real part of Eq. (20) reduces to

$$
-\gamma_{\mathbf{k}} E_{\mathbf{k}}^{+}=\pi \int_{-\infty}^{\infty}\left|T_{1,2}\right|^{2} E_{2}^{-}\left(E_{1}^{+}-E_{\mathbf{k}}^{+}\right) \delta_{\Delta \omega_{k 12}} \mathrm{~d} \mathscr{K}_{1,2}^{k},
$$

where $\gamma_{\mathbf{k}}$ is the total decrement caused by the nonlinear interactions.

In contrast to the DIA which operates with a two point two time correlation functions and/or their Fourier transforms $\left(Q^{ \pm}(\mathbf{k}, \tau)\right.$ and $\left.I_{\overline{\mathbf{k}}}^{ \pm}\right)$, the WTT implies Markovian closure and consequently operates with the Fourier transform of a two point one time correlation function $n_{\mathbf{k}}^{ \pm}(t)$ defined as

$$
\left\langle\phi_{\mathbf{k}}(t) \phi_{\mathbf{k}^{\prime}}^{*}(t)\right\rangle=n_{\mathbf{k}}^{ \pm}(t) \delta_{\mathbf{k}-\mathbf{k}^{\prime}} .
$$

As known, the nonlinear decrement of a one time correlation function for a zero time separation is twice larger then the nonlinear decrement of a two point correlation function since all temporal derivatives in the dynamic equations now act on both the time variables [21]. Consequently, according to Eq. (26) the dynamic equation for $n_{\mathbf{k}}^{+}(t)$ is

$$
\frac{\partial n_{\mathbf{k}}^{+}}{\partial t}=2 \pi \int_{-\infty}^{\infty}\left|T_{1,2}\right|^{2} n_{2}^{-}\left(n_{1}^{+}-n_{\mathbf{k}}^{+}\right) \delta_{\Delta \omega_{k 12}} \mathrm{~d} \mathscr{K}_{1,2}^{k} .
$$

Similar manipulations lead to the following equation for $n_{\mathbf{k}}^{-}(t)$

$$
\frac{\partial n_{\mathbf{k}}^{-}}{\partial t}=2 \pi \int_{-\infty}^{\infty}\left|T_{1,2}\right|^{2} n_{2}^{+}\left(n_{1}^{-}-n_{\mathbf{k}}^{-}\right) \delta_{\Delta \omega_{k 12}} \mathrm{~d} \mathscr{K}_{1,2}^{k} .
$$


These equations represent the WTT equations for weak MHD turbulence (with zero residual energy), which was first derived in Ref. [9] using the standard WTT technique.

Let us now turn back to the imaginary part of Eqs. (20). For validity of the WTT it is necessary that the frequency shift caused by nonlinear interactions is smaller than $\omega_{\mathbf{k}}$. Taking into account that, according to Eq. (21),

$$
\eta_{\mathbf{k}} \sim \frac{k_{\perp}^{4} E^{-}\left(0, k_{\perp}\right)}{\partial \omega_{\mathbf{k}} / \partial k_{z}}
$$

an analysis similar to the one performed above shows that, in addition to Eq. (25), the following condition should be satisfied

$$
\frac{\eta_{\mathbf{k}}}{\omega_{\mathbf{k}}} \ll 1
$$

\section{ANALYSIS OF THE WTT EQUATIONS}

The validity conditions of the WTT in a form similar to Eqs. (25) and (31) were first derived in Ref. [23] for drift wave turbulence. In that case, the validity criterium of the WTT was found to be identical to Eq. (31). The appearance of the additional condition (25) for the validity of the kinetic equation (28), is related to the degenerate character of the solution of the resonant condition $\omega_{\mathbf{k}}=\omega_{\mathbf{k}_{1}}-\omega_{\mathbf{k}_{2}}$, which implies $k_{2 z}=0$, and therefore requires the participation in nonlinear interactions of modes with very low frequencies (in the context of the performed analysis it is clear that $E^{-}\left(0, k_{\perp}\right)$ in Eq. (25) should be understood as the average energy density of the modes with $k_{z}<\eta_{\mathbf{k}} /\left(\partial \omega_{\mathbf{k}} / \partial k_{z}\right)$ and not the energy density of the mode with $k_{z} \equiv 0$ ). If Eq. (31) holds but the intensity of the low frequency modes is very low, such that left hand side of Eq. (25) is much greater then unity, then the kinetic equation (28) is not valid. However, it can be shown that the WTT is still valid then and the nonlinear interactions are dominated by four wave interactions. The corresponding kinetic equation was derived in Ref. [5]. If Eq. (31) is fulfilled and $\left(\eta_{\mathbf{k}} / \omega_{\mathbf{k}}\right) E^{-}\left(k_{z}, k_{\perp}\right) / E^{-}\left(0, k_{\perp}\right) \sim 1$, then the turbulence can still be called weak but no kinetic equation can be derived because in this case the contributions of three and four wave interactions in the nonlinear energy transfer are of the same order.

In the general case, the condition of validity of the WTT (31) does not contain the frequency but only its dispersive part $\left(\omega_{\mathbf{k}}-\mathbf{V}_{g} \cdot \mathbf{k}\right)$, because if there exists only one kind of waves, only the dispersive part of the frequency determines the width of the resonant area. Due to this reason, for waves with a quasi-linear dispersion relation, the application of the WTT requires the fulfillment of much stronger conditions for the amplitudes of the perturbations [24]. Alfvén waves are dispersiveless and the only reason why the WTT is applicable to incompressible MHD turbulence is the fact that nonlinear interaction is possible only between counter propagating waves (in Eq. (28), as is mathematically manifested by different signs of $\omega_{\mathbf{k}_{1}}$ and $\left.\omega_{\mathbf{k}_{2}}\right)$.

Introducing the characteristic velocity of the perturbations with a characteristic parallel length scale $l_{z} \sim 1 / k_{z}$ and perpendicular length scale $l_{\perp} \sim 1 / k_{\perp}$, respectively, as $v_{l}^{2} \sim k_{z} k_{\perp}^{2} E\left(k_{z}, k_{\perp}\right)$, the conditions (25) and (31) reduce to the following ones $k_{\perp} v_{l} / k_{z} V_{A} \ll 1$ and $\left[k_{\perp} v_{l} E\left(0, k_{\perp}\right)\right] /\left[k_{z} V_{A} E\left(k_{z}, k_{\perp}\right)\right] \ll 1$. Note that the first condition coincides with Eq. (3).

Another important temporal characteristic of the turbulence (together with $\tau_{a c}=1 / \eta_{\mathbf{k}}$ ) is the energy cascade timescale $\tau_{c a s}$ which represents the timescale of the energy cascade described by Eq. (28). For the energy transfer from one scale to another it is necessary that a wave packet should be totaly destroyed by nonlinear interactions. Consequently, the cascade timescale in heuristic models of the turbulence is also called the characteristic lifetime of the wave packet. Obviously, $\tau_{a c}<\tau_{c a s}$, or equivalently, the duration of a unit act of interaction among wave packets can not exceed its characteristic lifetime. A simple way of determining $\tau_{\text {cas }}$ is the following (a mathematically more sound derivation can be found in Ref. [20]): in the case of stationary turbulence, the left hand side of Eq. (28) is zero. But the characteristic timescale of the energy cascade can be determined if we retain only the modes with $\left|\mathbf{k}_{1 \perp}\right|>\left|\mathbf{k}_{\perp}\right|$ on the right hand side of Eq. (28). The equation then obtained describes the energy transfer from the mode with a wave number $\mathbf{k}_{\perp}$ to the modes with higher perpendicular wave numbers. The right hand side can be estimated as $n^{+} / \tau_{\text {cas }}$. The analysis of the left hand side terms similar to the one performed for the estimation of $\eta_{\mathbf{k}}$ yields

$$
\tau_{c a s} \sim \tau_{a c}
$$

and consequently, the WTT always suggests $N \sim\left(\tau_{c a s} / \tau_{a c}\right)^{2} \sim 1$. These arguments show that the IK model (as well as the heuristic model of the anisotropic turbulence described in the introduction, which will be considered in more 
details below), which implies $\tau_{a c} \sim\left(k V_{A}\right)^{-1}, \tau_{c a s} \sim V_{A} /\left(k v_{l}^{2}\right)$ and consequently $N \sim\left(V_{A} / v_{l}\right)^{2} \gg 1$, does not represent a model of weak MHD turbulence. In the next section we show that if isotropy is assumed then the WCA equations naturally lead to the IK spectrum in the strong turbulence limit. Note that in the considered isotropic case Eq. (3) leads to the (incorrect) conclusion that the turbulence is weak. On the other hand, because $\tau_{a c} k V_{A} \sim 1$ Eq. (31) yields that the IK model does not correspond to the weak turbulence limit.

As was shown above, the width of the resonant are is $\Delta k_{z} \sim\left(\eta_{\mathbf{k}} / \omega_{\mathbf{k}}\right) k_{z}$. Taking into account Eq. (31) this implies that physically nonlinear interactions in the framework of the WTT can be interpreted as resonant interactions among spatially very large wave packets [with characteristic size $\Delta l \sim\left(\omega_{\mathbf{k}} / \eta_{\mathbf{k}}\right) / k_{z}$ ]. Although the nonlinear interactions are weak, the interacting wave packets are very large such that an original wave packet decays before interacting wave packets pass through each other. Because the introduction of the Dirac delta function in Eq. (28) requires the limit $\eta_{\mathbf{k}} / \omega_{\mathbf{k}} \rightarrow 0$, it is sometimes stated that in the framework of the WTT the units of the nonlinear interactions are not wave packets of a finite spatial extent but spatially infinite Fourier harmonics [19].

Stationary solutions of the kinetic equations (28)-(29) can be found using the Zakharov transformation technique $[20,9]$. Here we use a different approach. According to Eqs. (28)-(29) if $n^{ \pm}\left(0, k_{\perp}\right)$ is the solution of stationary equation, then $n^{ \pm}\left(k_{z}, k_{\perp}\right)=f\left(k_{z}\right) n^{ \pm}\left(0, k_{\perp}\right)$ where $f\left(k_{z}\right)$ is any function, is also the solution [9]. Noting also that in the stationary case $n^{ \pm}\left(k_{z}, k_{\perp}\right) \equiv E^{ \pm}\left(k_{z}, k_{\perp}\right)$ and using Eqs. 30) and (32), we get

$$
\tau_{c a s} \sim \frac{f\left(k_{z}\right) V_{A}}{k_{\perp}^{4} \mathscr{E}\left(k_{z}, k_{\perp}\right)}=\frac{f\left(k_{z}\right) k_{z} V_{A}}{k_{\perp}^{2} v_{l}^{2}} .
$$

Using the standard relation $\varepsilon \sim v_{l}^{2} / \tau_{\text {cas }}$ we obtain

$$
\mathscr{E}\left(k_{z}, k_{\perp}\right) \sim\left[\frac{\varepsilon f\left(k_{z}\right) V_{A}}{k_{z}}\right]^{1 / 2} k_{\perp}^{-3} .
$$

The energy spectrum and cascade time given by Eqs. (33) and (34) coincides with the results of the heuristic model described in the introduction, whereas these models yield a different autocorrelation time $\tau_{a c}$. The latest is a very important characteristic of the turbulence and contributes in the determination of such characteristics of the turbulent system as the transport coefficients [25] and the frequency spectrum [26]. In fact, the heuristic model is not realizable neither in the WTT nor in the strong turbulence regime because it is based on two controversial assumptions: on the one hand, it assumes a purely perpendicular cascade, i.e., it is based on three wave resonant interactions of the WTT. On the other hand, it is supposed that the energy cascade needs many collisions between counter propagating wave packets $(N \gg 1)$, which is incompatible with the WTT. The main physical reason of the failure is the following: the heuristic model implicitly assumes that the longitudinal size of wave packet is of the order $L_{z} \sim 1 / k_{z}$, i.e., it is assumed that $\Delta k_{z} / k_{z} \sim 1$ where $\Delta k_{z} \sim 1 / L_{z}$ is the longitudinal width of the wave packet in the Fourier space. Consequently, it is assumed that the time necessary for wave packets to pass through each other is $\tau_{a c}=L_{z} / V_{A} \sim\left(k_{z} V_{A}\right)^{-1}$. Actually, as it was shown above in the framework of the WTT, wave packets have a much larger spatial extent $\Delta k_{z} / k_{z} \sim \eta_{\mathbf{k}} / \omega_{\mathbf{k}} \ll 1$. Note that again Eqs. (3) and (31) yield different results for the heuristic model: according to Eq. (3) the turbulence is weak, whereas according to Eq. (31) the model does not correspond to the weak turbulence regime.

\section{STRONG MHD TURBULENCE}

In this section, we consider the strong turbulence limit which implies

$$
\frac{\eta_{\mathbf{k}}}{\omega_{\mathbf{k}}}>1
$$

In the framework of the WCA this implies the neglection of the second term in the denominators of Eqs. (22) and (23). Namely, integrating both sides of Eq. (20) with respect to $\omega$, we note that in the stationary case the left hand side of the obtained equation vanishes. Therefore, the dropped dissipation term should be restored. Dropping the frequency containing terms with respect to the terms containing $\eta_{\mathbf{k}}$, we finally obtain (details of the derivation can be found in Ref. [17])

$$
\eta_{\mathbf{k}}=\int_{-\infty}^{\infty}\left|T_{1,2}\right|^{2} \frac{E_{\mathbf{k}_{2}}}{\eta_{\mathbf{k}_{1}}+\eta_{\mathbf{k}_{2}}} \mathrm{~d} \mathscr{K}_{1,2}^{k}
$$




$$
\bar{v} k^{2} E_{\mathbf{k}}=\int_{-\infty}^{\infty}\left|T_{1,2}\right|^{2} \frac{E_{\mathbf{k}_{2}}\left(E_{\mathbf{k}_{1}}-E_{\mathbf{k}}\right)}{\eta_{\mathbf{k}}+\eta_{\mathbf{k}_{1}}+\eta_{\mathbf{k}_{2}}} \mathrm{~d} \mathscr{K}_{1,2}^{k}
$$

Suppose the turbulence is isotropic, i.e., assume $E_{\mathbf{k}} \equiv E_{k}$ and $\eta_{\mathbf{k}} \equiv \eta_{k}$. Multiplying Eq. (37) by $4 \pi k^{2}$ and integrating from some $k$ in the inertial interval to infinity, and noting that the obtained expression on the left hand side represents the dissipation rate of the turbulence, we obtain

$$
\varepsilon=4 \pi \int_{k}^{\infty} k^{4} \mathrm{~d} k \int_{-\infty}^{\infty} \frac{\left|T_{1,2}\right|^{2} E_{k_{2}}\left(E_{k_{1}}-E_{k}\right)}{\eta_{k}+\eta_{k_{1}}+\eta_{k_{2}}} \mathrm{~d} \mathscr{K}_{1,2}^{k}
$$

To study the scaling properties of the turbulence, we seek for the inertial range a solution of Eqs. (36) and (38) in the form $E_{k}=A k^{m}$. Substituting these expressions into Eq. (36) and taking into account that for $k \gg k_{2},\left|T_{1,2}\right| \sim k$, we see that the integral on the right hand side of Eq. (36) diverges at $k_{2} \rightarrow 0$ if $m<-3$. Note that $m>-3$ implies that the characteristic value of the perturbation $v_{l} \sim\left(k^{3} E_{k}\right)^{1 / 2}$ is increasing when $k$ increases. Due to the condition $\varepsilon \sim v_{l}^{2} / \tau_{c a s}$ this means that the cascade timescale decreases in the same limit, which normally does not take place (for the Kolmogorov spectrum $m=-11 / 3$ ). Therefore, the integral in Eq. (36) is dominated by the low wave number modes. But as it was mentioned above, before applying the WCA closure scheme the velocity field of the low frequency modes is considered to be removed by the Galilean transformation. Therefore, the energy of the low frequency modes $E_{k}$ contains only the magnetic part of the low frequency mode energy. The magnetic field of theses modes form a background field for the high frequency modes in the inertial interval.

Using a delta function to integrate over $k_{1}$ in Eq. (36) and noting that $\left|\mathbf{k}-\mathbf{k}_{2}\right| \approx k$, we obtain $\eta_{k} \sim k^{2} \int E_{k_{2}} \mathrm{~d}^{3} \mathbf{k}_{2} / \eta_{k} \sim$ $k^{2} V_{A}^{2} / \eta_{k}$, and consequently, $\eta_{k} \sim k V_{A}$.

In contrast to Eq. (36), due to the presence of the multiplier $\left(E_{k_{1}}-E_{k}\right)$, the integrals in Eq. (38) are convergent for the small values of $k_{2}$ when $m>-4$. Consequently, noting that the main contribution in the integral comes from the wave numbers with $k_{1} \sim k_{2} \sim k$ we obtain $\varepsilon \sim A^{2} k^{8+2 m} / \eta_{k}$. For the one dimensional energy spectrum $\left(\mathscr{E}_{k}=4 \pi^{2} E_{k}\right)$ this yields $\mathscr{E}_{k} \sim\left(\varepsilon V_{A}\right)^{1 / 2} k^{-3 / 2}$, which represents the IK spectrum. Therefore, it was shown that if isotropy is assumed then the WCA equations automatically lead to the IK model in the strong turbulence limit.

The anisotropic solutions of the WCA Eqs. (20)-(21) were studied in Ref. [17]. It has been shown that there exist two stationary solutions. The first one represents the Goldreich-Sridhar [6] model with $\overline{\mathscr{E}}_{k_{\perp}} \sim \varepsilon^{2 / 3} k_{\perp}^{-5 / 3}$ (where $\overline{\mathscr{E}}_{k_{\perp}}$ is the one dimensional perpendicular energy spectrum). If there exist a strong external magnetic field $\mathbf{B}_{0}$ and the turbulence is excited at some scale with a characteristic magnetic field value $\bar{b}_{0}$ (in velocity units), then the second solution is $\overline{\mathscr{E}}_{k_{\perp}} \sim\left(\varepsilon \bar{b}_{0}\right)^{1 / 2} k_{\perp}^{-3 / 2}$, in agreement with the results observed in direct numerical simulations of incompressible MHD turbulence $[10,12,13]$ in the presence of a strong external magnetic field.

\section{CONCLUSIONS}

Both strong and weak turbulence regimes of incompressible MHD turbulence were considered in the framework of the WCA. We derived a general validity condition for the WTT given by Eq. (31). We showed that in the framework of the WTT the autocorrelation and cascade timescales are always of the same order of magnitude and, consequently, the WTT always suggests $N \gg 1$. Physically this is caused by the fact that the framework of the WTT can be interpreted as the resonant interaction among spatially very large wave packets [with characteristic size $\Delta l \sim\left(\omega_{\mathbf{k}} / \eta_{\mathbf{k}}\right) / k_{z}$ ]. Although the nonlinear interactions are weak, the interacting wave packets are very large such that an original wave packet decays before interacting wave packets pass through each other. We also considered the strong turbulent limit and we showed that if isotropy is assumed then the WCA equations automatically lead to the IK model.

\section{ACKNOWLEDGMENTS}

G.G. and S.M.M. acknowledge the hospitality of the Abdus Salam International Center for Theoretical Physics (ICTP) where part of the work was done. G.G. acknowledges partial support from Georgian NSF grant ST07/4-193. These results were obtained in the framework of the projects GOA/2009-009 (K.U.Leuven), G.0304.07 (FWO-Vlaanderen) and C 90347 (ESA Prodex 9). Financial support by the European Commission through the SOLAIRE Network (MTRN-CT-2006-035484) is gratefully acknowledged. 


\section{REFERENCES}

1. P. S. Iroshnikov, Astron. Zh. 40, 742 (1963).

2. R. H. Kraichnan, Phys. Fluids 8, 1385-1387 (1965).

3. J. V. Shebalin, W. H. Matthaeus and D. Montgomery, J. Plasma Phys. 29, 525-547 (1983).

4. J. C. Higdon, Astrophys. J. 285, 109-123 (1984).

5. S. Sridhar and P. Goldreich, Astrophys. J. 432, 612-621 (1994).

6. P. Goldreich and S. Sridhar, Astrophys. J. 438, 763-775 (1995).

7. C. S. Ng and A. Bhattacharjee, Phys. Plasmas 4, 605-610 (1997).

8. J. Cho and E. Vishniac, Astrophys. J. 539, 273-282 (2000).

9. S. Galtier, S. V. Nazarenko, A. C. Newell, and A. Pouquet, J. Plasma Phys. 63, 447-488 (2000).

10. J. Maron and P. Goldreich, Astrophys. J. 554, 1175-1196 (2001).

11. J. Cho, A. Lazarian and E. Vishniac, Astrophys. J. 564, 291-301 (2002).

12. W.-C. Müller, D. Biskamp and R. Grappin, Phys. Rev. E 67, 066302 (2003).

13. W.-C. Müller and R. Grappin, Phys. Rev. Lett. 95, 114502 (2005).

14. S. Oughton and W. H. Matthaeus, Nonlin. Proc. in Geophysics 12, 299-310 (2005).

15. A. Schekochihin, and S. C. Cowley, "Turbulence and Magnetic Fields in Astrophysical Plasmas," in Magnetohydrodynamics:

Historical Evolution and trends, edited by S. Molokov et al., Springer, Dordrecht, 2007, p. 85. astro-ph/0507686.

16. A. Beresnyak and A. Lazarian, Astrophys. J. 640, L175-L178 (2006).

17. G. Gogoberidze, Phys. Plasmas 14, 022304-11 (2007).

18. A. Beresnyak and A. Lazarian, Astrophys. J. 682, 1070-1075 (2008).

19. B. B. Kadomtsev, Plasma Turbulence, Academic, New York, 1965, pp. 54-55.

20. V. E. Zakharov, V. S. L'vov and G. Falkovich, Kolmogorov Spectra of Turbulence I, Springer-Verlag, Berlin, 1992, pp. 135-137.

21. D. C. Leslie, Developments in the Theory of Turbulence, Oxford University Press, 1973, pp. 24-93.

22. R. H. Kraichnan, J. Fluid Mech. 5, 497-543 (1959).

23. M. Ottaviani and J. Krommes, Phys. Rev. Lett. 69, 2923-2926 (1992).

24. V. S. L'vov, 1, Yu. L'vov, A. C. Newell, and V. Zakharov, Phys. Rev. E 56, 390-405 (1997).

25. J. A. Krommes, Phys. Reports 360, 1-352 (2002).

26. Y. Kaneda, Phys. Fluids A 5, 2835-2845 (1993). 